\title{
ABSTRACTS
}

\section{SCIENTIFIC ORAL PRESENTATIONS}

\section{June 2014}

\author{
S1 - Session1 0930-0945 \\ doi:10.1017/cjn.2014.45
}

Identification of the azole class of antifungals as potent inhibitors of glioblastoma growth and tumour metabolism (Young Investigator Award Winner - Basic/Translational Science)

\section{S. Agnihotri, K. Burrell, S.K. Singh, A.Vartanian, K. Aldape, G.} Zadeh

Arthur and Sonia Labatt Brain Tumour Research Centre, The Hospital for Sick Children, Toronto, Ontario

Rapidly proliferating tumour cells preferentially use aerobic glycolysis over oxidative phosphorylation (OXPHOS) to support growth and survive unfavorable microenvironment conditions. This metabolic reprogramming is referred to as the Warburg effect and offers a novel way to target cancer cells. We previously demonstrated that the glycolytic enzyme hexokinase 2 (HK2) is crucial for the Warburg effect in human glioblastoma multiforme (GBM), the most common malignant brain tumor. HK2 has little to no expression in normal brain making it an attractive target for targeting the Warburg effect. However, no direct inhibitor of HK2 exists so we explored whether a system biology approach to identify gene networks regulated by or associated with HK2 that could lead to promising treatment strategies. Using HK2 knockdown by siRNA in established GBM cell lines and primary GBM cultures we established gene signatures and networks associated with HK2 expression, identifying over 1000 genes with a 2 fold change with $p$-value $<0.01$. Loss of HK2 led to attenuation of several pro GBM signaling pathways affecting tumour cell invasion, glucose metabolism and proliferation. Using a small drug screen we identified the azole class of antifungals as inhibitors of tumour metabolism by reducing proliferation, lactate production, glucose uptake in GBM cells but not primary normal human astrocytes or normal neural stem cells. Interestingly, several antifungal Azole compounds were more potent at killing GBM cells in hypoxic conditions. Current work is focused on the in vivo efficacy of these azole compounds in pre-clinical orthotopic xenograft mouse models and transgenic models of GBM. In summary, the azole class of antifungals may represent a new way of targeting tumour metabolism in tumours dependent on aerobic glycolysis.
S2 - Session1 0945-1000

doi:10.1017/cjn.2014.46

CIC in neurodevelopment and oligodendroglioma

AD Rogers ${ }^{l}, R$ Dixit $^{2}$, SO Lawn ${ }^{2}, S \mathrm{Li}^{3}, C J$ Schuurmans ${ }^{3}, J G$ Cairncross $^{\text {, JA }}$ Chan $^{2}$

Departments of Clinical Neuroscience ${ }^{1}$, Pathology \& Laboratory Medicine ${ }^{2}$ and Biochemistry \& Molecular Biology ${ }^{3}$, University of Calgary, Calgary, Alberta

Oligodendrogliomas (ODG) are distinctive brain tumours composed of cells resembling oligodendrocyte precursor cells (OPCs). Genetic hallmarks of ODGs include $1 \mathrm{p} / 19 \mathrm{q}$ chromosomal co-deletion and IDH1/2 mutation. Recently, the gene encoding Capicua (CIC), on chr19q13.2, was identified as mutated in most ODGs with concurrent $1 \mathrm{p} / 19 \mathrm{q}$ loss and IDH1/2 mutation a genetic signature rare in other cancers. Mutation of the retained $19 \mathrm{q}$ CIC allele is likely functionally important, but how it contributes to ODG biology is unknown. The aims of this study are to characterize the temporal and spatial expression of $\mathrm{Cic}$ in the normal mouse cerebrum, and to determine if CIC loss affects proliferation or differentiation of neural progenitors. To examine CIC expression, immunofluorescence staining was performed on forebrain tissue over a developmental timecourse. CIC biologic functions were determined using a loss-of-function approach, introducing CIC shRNA or control shRNA into neural progenitors. Cells were examined for proliferation, and for cell identity using a panel of markers. Our data supports a role for Cic in regulating several processes in neural progenitors that are relevant to cancer including proliferation and, possibly, differentiation. CIC loss due to mutational inactivation may thus deregulate processes relevant to oligodendrogliomagenesis.

\section{S3 - Session1 1000-1015}

doi:10.1017/cjn.2014.47

Targeting Glioblastoma Invasion with GSK-3 inhibitors: Rapid Effects on the EMT Marker Vimentin

Michal O. Nowicki ${ }^{l}$, E. Antonio Chiocca ${ }^{l}$, Sean E. Lawler

Harvey Cushing Neuro-oncology Laboratories, Department of Neurosurgery, Brigham and Women's Hospital, Harvard Medical School, Boston, MA, USA.

Tumor cell invasion of surrounding normal brain remains a formidable obstacle to effective glioblastoma therapy. We previously identified that inhibitors of the protein kinase GSK-3 